\title{
Endothelial Progenitor Cell Dysfunction as a Biomarker of Cardiovascular Risk in Obese Patients
}

\author{
Alexander E. Berezin \\ Consultant of Therapeutic Unit, Internal Medicine Department, \\ State Medical University of Zaporozhye, Ukraine.
}

\begin{abstract}
Obesity is common endocrine disorders that is characterized an increased prevalence worldwide for last decades. Based on the Adult Treatment Panel-III criteria subjects with established obesity and co-existing other metabolic abnormalities including dyslipidemia, insulin resistance (IR), increased fasting glucose and impaired glucose tolerance, are referred metabolically non-healthy, whereas obese individuals without these abnormalities might be defined as metabolically healthy. However, the risk stratification in both obesity phenotype patients is not accurately determined. Formerly endothelial progenitor cells (EPCs) were found a player in target organ damage at the different stages of obese. Probably, EPCs' dysfunction defined as lowered number and / or weak function of EPCs as a biomarker of altered endothelial function might be a predictor of cardiovascular $(\mathrm{CV})$ risk in obese patients The aim of the short commentary is to summarize our knowledge regarding the role of endothelial progenitor cell dysfunction in prognostication of $C V$ events in patients with established obesity.
\end{abstract}

Keywords: obesity; metabolically healthy obesity; cardiovascular risk; stratification; endothelial progenitor cells.

Obesity is common endocrine disorders, a prevalence of which progressively arises worldwide for last decades [1]. Based on the Adult Treatment Panel-III (ATP-III) criteria subjects with established obesity and co-existing other metabolic abnormalities including dyslipidemia, insulin resistance (IR), increased fasting glucose and impaired glucose tolerance, are referred metabolically non-healthy, whereas obese individuals without these abnormalities might be defined as metabolically healthy [2]. Interestingly, there is not strong definition of metabolically healthy obese as a transient age- and ethnic-related phenotype accompanied to some behavioral and environmental factors [3]. However, lack of follow sings i.e. abdominal type of obesity, IR, impaired fasting glucose / glucose tolerance, and low level of cardiorespiratory fitness, is considered plenty acceptable criteria of metabolically healthy obese [4]. Thus, there are at least two main body-size phenotypes (metabolically healthy and metabolically non-healthy) of individuals with established obesity [5], but the cardiometabolic risk amongst these patients' populations extremely distinguishes [6].

The majority of obese patients, who were involved in the large longitudinal studies, have been qualified neither metabolically non-healthy individuals nor patients with abdominal obesity and metabolic syndrome / type two diabetes mellitus (T2DM). Dramatically raise of the risk of cardiovascular (CV) incidences in metabolically non-healthy obese is now well known [7]. In contrast, other metabolically healthy obese individuals, for whose mild-to-moderate increase of CV risk is uncertainly established, were discovered not a bit carefully. Whether the same prognostication models would be effective to much more accurate stratify individuals with both obese phenotypes at higher CV risk is not fully understood. Consequently, a risk prediction of CV events and CV diseases for both obese phenotypes' patients might base on different biomarkers and distinguished frequently pragmatic approaches $[8,9]$.

The contemporary "fat-but-fit" hypothesis has issued from that the metabolically healthy obese is a transient state, which may translate into a metabolically active state over time affecting endogenous reparative response especially in the endothelium [10-12]. In this context, endothelial progenitor cell (EPC) dysfunction may play a pivotal role in target organ damage at the different stages of obesity $[13,14]$. 
EPCs have defined as cells, which are positively labeled with both hematopoietic stem cells (CD34) and endothelial cell markers (vascular endothelial growth factor receptor-2 [VEGFR2] and CD31) cumulatively [15]. Later an expression of other hematopoietic stem cells markers (CD133, AC133) and some endothelial markers (platelet-endothelial cell adhesion molecule known as CD31, VEcadherin also known as CD 144, caveolin-1, von Willebrand factor, and endothelial NO synthase) on the surface of EPCs was found [16]. Therefore, some subsets of EPCs may express mononuclear antigens, i.e. CD14, CD11b, CD11c, together with CD34 or VEGFR2, CD45, Tei2 and Flt-1 [17] and shape so called "non-classical" phenotypes. All these EPCs remain vascular protective capacity and may differentiate into mature endothelial cells under effect of microenvironment, paracrine regulators and appropriate growth factors (e.g., VEGF, fibroblast growth factor).

Additionally, there are at least two types of EPCs labelled as early outgrowth EPCs and late outgrowth EPCs and isolated from similar source [18]. Both subpopulations of EPCs have expressed CD144, Flt-1, KDR (VEGFR2), and CD45 markers in different manner. Late outgrowths EPCs produced more nitric oxide, incorporated more into human umbilical vein ECs monolayer, and are able to better form capillary tube than early EPC [19]. Early EPC secreted more pro-angiogenic cytokines (VEGF and interleukin-8) than late EPC at culture [20]. Moreover, early EPCs intervened in the monolayer of human umbilical vein endothelial cells (HUVEC), but more late EPCs were incorporated to HUVEC [21]. Overall both subpopulations of EPC might distinguish one another in ability to differentiate into circulating angiogenic cells (referred as early EPCs), shaping endothelial colony cells (referred as late outgrowth EPCs), and inducing vasculogenesis [22]. Whether early outgrowth and late outgrowth EPCs mediate similar effect on vascular protection and tissue repair is uncertain [23].

Increased adipocyte size is hypothesized to signal the recruitment of various types of progenitor cells including endothelial progenitor cells [24]. In metabolically healthy obese individuals the number of EPCs in the circulation is frequently increased or near normal [25]. In contrast, development of metabolically non-healthy obese associates with the reduced ability of EPCs to realize their potency in proliferation, differentiation, adhesion, migration, incorporation into tubular structures, and survival is now defined as EPC dysfunction [26]. The wear EPCs functionality may associate with lowering EPCs' count in the peripheral blood that is considered an initiation of endothelial dysfunction [27]. Nevertheless, EPCs dysfunction well predicts CV risk in general population and in subjects with established CV and metabolically non-healthy obese [28].

The primary reason of deficiency of circulating EPCs' number in metabolically non-healthy obese is not fully clear. In fact, glucose toxicity, lipid toxicity, inflammation and reactive oxidative species are now recognized as mainly factors contributing in EPC dysfunction in diabetes [29]. They act through decreased expression of protein kinase A regulatory subunit $1 \beta$ (PRKAR $1 \beta$ ), activation of protein kinase A (PKA), matrix metalloproteinase-9, and phosphorylation of $\alpha 4$ integrin on serine 988 [30]. However, alteration of structure / function and reduced number of circulating EPCs has now identified in prediabetes [31]. In contrast, controversial results regarding being of progenitor dysfunction in obese individuals beyond diabetes were found within last decade [32]. The first controversial affects the metabolically non-healthy obese in children and adolescents, in which circulating EPC count is elevated accompanying to BMI that the metabolically non-healthy obese adults may present exaggerated numbers of endothelial cell-originated microparticles, and low number of EPCs, and high levels of adipokines in peripheral blood beyond inflammatory condition [33]. Moreover, in adult metabolically non-healthy obese individuals circulating EPC number may decrease along with elevated serum level of visfatin, insulin resistance and accumulation of oxidative stress product [3436].

Recent studies have found that the deficiency of EPC and their functional alterations strongly associated with the development and progression of CV disease [43, 44]. Consequently, dysfunction of EPC might be early and probably potentially reversible stage of exhaustion of endogenous endothelial reparation mechanisms leading to the development of endothelial dysfunction and asymptomatic vascular damage. Whether EPC dysfunction would be early biomarker in obese to risk stratification is not completely understood, while this suggestion is obviously promised.

In conclusion, it is extremely difficult to plenty accurate determine the predictive value of EPC dysfunction in obese depending phenotypes, while observational studies have shown the strong difference between weak functionality and low number of EPC and CV risk. Large clinical 
investigations are required to explain in detail whether progenitor dysfunction is not only whiteness of nature evolution of the obese, but it is factor contributing in transformation of healthy obesity to metabolically non-healthy phenotype.

Conflict of interests: no significant financial conflicts of interest relevant to the article topic is declared

\section{References}

[1] Vanuzzo D, Pilotto L, Mirolo R, Pirelli S. Cardiovascular risk and cardiometabolic risk: an epidemiological evaluation. G Ital Cardiol (Rome). 2008; 9(4 Suppl 1): 6S-17S.

[2] Rey-López JP, de Rezende LF, Pastor-Valero M, Tess BH. The prevalence of metabolically healthy obesity: a systematic review and critical evaluation of the definitions used. Obesity Reviews. 2014; 15 (10): 781-90

[3] Global Burden of Metabolic Risk Factors for Chronic Diseases Collaboration (BMI Mediated Effects), Lu Y, Hajifathalian K, Ezzati M, Woodward M, Rimm EB, Danaei G. Metabolic mediators of the effects of body-mass index, overweight, and obesity on coronary heart disease and stroke: a pooled analysis of 97 prospective cohorts with 1.8 million participants. Lancet. 2014; 383(9921): 970-83.

[4] Gregorio-Arenas E, Ruiz-Cabello P, Camiletti-Moirón D, Moratalla-Cecilia N, Aranda P, LópezJurado $\mathrm{M}$, et al. The associations between physical fitness and cardiometabolic risk and bodysize phenotypes in perimenopausal women. Maturitas. 2016; 92: 162-7.

[5] Eckel RH, Cornier MA. Update on the NCEP ATP-III emerging cardiometabolic risk factors. BMC Med. 2014; 12:115.

[6] Li Z, Guo X, Liu Y, Zhang N, Chang Y, Chen Y, et al. Metabolism rather than obesity is associated with ischemic stroke: a cross-sectional study in rural Northeastern China. Springerplus. 2016; 5(1): 1419.

[7] Blüher M. The distinction of metabolically 'healthy' from 'unhealthy' obese individuals". Current Opinion in Lipidology. 2010; 21 (1): 38-43.

[8] Berezin A. Cardiac biomarkers in diabetes mellitus: new dawn for risk stratification? Diabetes \& Metabolic Syndrome: Clinical Research \& Reviews. 2016 [epub ahead of print] Doi: 10.1016/j.dsx.2016.12.032.

[9] Berezin A. Biomarkers for cardiovascular risk in diabetic patients. Heart. 2016; 102: 1939-1941.

[10] Bluher M. Mechanisms in endocrinology: Are metabolically healthy obese individuals really healthy? Eur J Endocrin. 2014; 171 (6): R209-R219.

[11] Stefan N, Häring HU, Hu FB, Schulze MB. Metabolically healthy obesity: epidemiology, mechanisms, and clinical implications. Lancet Diabetes Endocrinol. 2013; 1: 152-162.

[12] Duncan GE. The "fit but fat" concept revisited: population-based estimates using NHANES. Int J Behav Nutr Phys Act. 2010; 7:47.

[13] Berezin A. Endothelial progenitor cells dysfunction and impaired tissue reparation: the missed link in diabetes mellitus development. Diabetes \& Metabolic Syndrome: Clinical Research \& Reviews. 2016 [ahead of print] Doi: 10.1016/j.dsx.2016.08.007

[14] Berezin A. Progenitor endothelial cell dysfunction in obese patients: possibilities for cardiovascular risk prediction. Journal of Clinical \& Experimental Cardiology. 2016; 7 (10): 148-150.

[15] Asahara T, Murohara T, Sullivan A, Silver M, van der Zee R, Li T. et al. Isolation of putative progenitor endothelial cells for angiogenesis. Science. 1997; 275:964-967

[16] Peichev M, Naiyer AJ, Pereira D, Zhu Z, Lane WJ, Williams M et al. Expression of VEGFR-2 and $\mathrm{AC} 133$ by circulating human $\mathrm{CD} 34(+)$ cells identifies a population of functional endothelial precursors. Blood. 2000;95:952-958

[17] Abe Y, Ozaki Y, Kasuya J, Yamamoto K, Ando J, Sudo R, Ikeda M, Tanishita K. Endothelial progenitor cells promote directional three-dimensional endothelial network formation by secreting vascular endothelial growth factor. PLoS One. 2013; 8(12):e82085.

[18] Hur J, Yoon CH, Kim HS, Choi JH, Kang HJ, Hwang KK. et al. Characterization of two types of endothelial progenitor cells and their different contributions to neovasculogenesis. Arterioscler Thromb Vasc Biol. 2004; 24: 288-293. 
[19] Lin Y, Weisdorf DJ, Solovey A, Hebbel RP. Origins of circulating endothelial cells and endothelial outgrowth from blood. J Clin Invest. 2000;105:71-77

[20] Schmeisser A, Garlichs CD, Zhang H, Eskafi S, Graffy C, Ludwig J, et al. Monocytes coexpress endothelial and macrophagocytic lineage markers and form cord-like structures in Matrigel under angiogenic conditions. Cardiovasc Res. 2001; 49(3): 671-80.

[21] Friedrich EB, Walenta K, Scharlau J, Nickenig G, Werner N. CD34-/CD133+/VEGFR-2+ endothelial progenitor cell subpopulation with potent vasoregenerative capacities. Circ Res. 2006; 98: e20-e25.

[22] Fadini GP, Avogaro A. Potential manipulation of endothelial progenitor cells in diabetes and its complications. Diabetes Obes Metab. 2010; 12(7): 570-83.

[23] Berezin A. Epigenetic Mechanisms of Endothelial Progenitor Cell Dysfunction. J Clin Epigenet. 2016, 2 (2): 24-26

[24] Asahara T. Endothelial progenitor cells for vascular medicine. Yakugaku Zasshi. 2007; 127(5):841-5.

[25] Berezin A. "Impaired Phenotype" of Endothelial Cell-Derived Microparticles: Causality Factor Contributed the "Vascular Competence" in Diabetes and Metabolic Syndrome? Diabetes Res Treat. 2016; 3: 133-136.

[26] Asahara T, Murohara T, Sullivan A, Silver M, van der Zee R, Li T, et al. Isolation of putative progenitor endothelial cells for angiogenesis. Science. 1997;275:964-967

[27] Fadini GP, de Kreutzenberg SV, Coracina A, Baesso I, Agostini C, Tiengo A, et al. Circulating CD34+ cells, metabolic syndrome, and cardiovascular risk. Eur Heart J 2006; 27: 2247-2255.

[28] Berezin AE, Kremzer AA, Berezina TA, Martovitskaya YV, Gronenko EA. Association between serum osteoprotegerin level and numerous of circulating endothelial- derived and mononuclearderived progenitor cells in patients with metabolic syndrome. Data in Brief. 2016; 8: 717-722.

[29] Berezin AE, Samura TA, Kremzer AA, Berezina TA, Martovitskaya YV, Gromenko EA. An association of serum vistafin level and number of circulating endothelial progenitor cells in type 2 diabetes mellitus patients. Diabetes Metab Syndr. 2016. doi: 10.1016/j.dsx.2016.06.008. [Epub ahead of print].

[30] Berezin AE. Metabolic memory phenomenon in diabetes mellitus: Achieving and perspectives. Diabetes Metab Syndr. 2016; 10(2 Suppl 1):S176-83.

[31] Abplanalp WT, Conklin DJ, Cantor JM, Ginsberg MH, Wysoczynski M, Bhatnagar A, et al. Enhanced Integrin $\alpha 4 \beta 1$-Mediated Adhesion Contributes to a Mobilization Defect of Endothelial Progenitor Cells in Diabetes. Diabetes. 2016 [Epub ahead of print]

[32] Fadini GP, Sartore S, Agostini C, Avogaro A. Significance of endothelial progenitor cells in subjects with diabetes. Diabetes Care 2007; 30: 1305-1313.

[33] Pires A, Martins P, Paiva A, Pereira AM, Marques M, Castela E, et al. Circulating endothelial progenitor cells in obese children and adolescents. J Pediatr (Rio J). 2015; 91(6):560-6.

[34] Noci MV, Ramírez R, Lluch M, Rodríguez M, Carracedo J. Changes in endothelial microparticles and endothelial progenitor cells in obese patients in response to surgical stress. $\mathbf{J}$ Bone Joint Surg Am. 2015; 97(5): 353-8.

[35] Chen S, Sun L, Gao H, Ren L, Liu N, Song G. Visfatin and oxidative stress influence endothelial progenitor cells in obese populations. Endocr Res. 2015; 40(2): 83-7.

[36] Bochenek ML, Schütz E, Schäfer K. Endothelial cell senescence and thrombosis: Ageing clots. Thromb Res. 2016; 147:36-45. 